PROFILES OF RATIONAL NUMBER KNOWLEDGE

\title{
Profiles of rational number knowledge in Finnish and Flemish students - A Multigroup Latent Class Analysis
}

\author{
Jake McMullen \\ Department of Teacher Education and Centre for Learning Research, University of Turku, \\ Finland
}

Jo Van Hoof, Tine Degrande, Lieven Verschaffel, \& Wim Van Dooren

Centre for Instructional Psychology and Technology, KU Leuven, Belgium

Correspondence concerning this article should be addressed to Jake McMullen, Centre for Learning Research, Turun Yliopisto, 20014, Finland. Email: jake.mcmullen@utu.fi

Manuscript accepted for publication in Learning and Individual Differences, February 10, 2018. DOI:10.1016/j.lindif.2018.02.005

https://www.sciencedirect.com/science/article/pii/S1041608018300293 


\title{
PROFILES OF RATIONAL NUMBER KNOWLEDGE
}

\begin{abstract}
Students have a great deal of difficulties learning about rational number concepts, as they are confounded by misapplying reasoning about natural numbers to fractions and decimals, referred to as a natural number bias. For example, students often think that the number of digits of a decimal, or the size of the component numbers of fractions, is enough information to determine the magnitude of rational numbers. As well, students have trouble understanding that there is an infinite number of numbers between any two fractions or decimals. Using multigroup latent class analysis, the present study examines the structure of 611 Finnish and Flemish students' rational number knowledge in order to determine the similarities and differences between these two sub-samples. Results reveal that, while the Flemish students performed somewhat better, there were only relatively minor differences in the structure of the two sub-samples' rational number knowledge. In general, it appears that the natural number bias affects these Finnish and Flemish students' knowledge of the size and density of fractions and decimals in similar ways.
\end{abstract}




\section{PROFILES OF RATIONAL NUMBER KNOWLEDGE}

\section{Introduction}

Learners - in wide age ranges and across nationalities - face difficulties when learning about rational numbers (Torbeyns, Schneider, Xin, \& Siegler, 2015; Vamvakoussi, Christou, Mertens, \& Van Dooren, 2011). These difficulties are especially troubling given the importance of rational numbers in both later mathematical learning (DeWolf, Bassok, \& Holyoak, 2015; Siegler et al., 2012) and worklife activities (Handel, 2016). Part of these difficulties are accounted for by difficulties in the transition from reasoning about numbers as being natural number exclusively, to numbers including also rational numbers (Ni \& Zhou, 2005). While natural number knowledge can often be used to reason successfully about rational numbers, they also have some features that are different from natural numbers and thus an overreliance on natural number reasoning has been identified as a major cause of difficulties with rational number learning (Vamvakoussi, Van Dooren, \& Verschaffel, 2012). This natural number bias has been identified in a large number of instances (e.g., Kainulainen, McMullen, \& Lehtinen, 2017; Nunes \& Bryant, 2008; Obersteiner \& Tumpek, 2016; Vamvakoussi et al., 2011; Van Hoof, Janssen, Verschaffel, \& Van Dooren, 2015). While the universality of such a bias across educational contexts has been examined with one particular feature of the bias, namely the density of the set of rational numbers (Vamvakoussi et al., 2011), this has never been done on a holistic level. The present study aims to examine, in distinct samples of Finnish and Flemish children, the extent to which there are similarities and differences in the over-application of natural number concepts when reasoning about multiple aspects of rational numbers across the two sub-samples.

\subsection{Rational number conceptual knowledge and the natural number bias}

Many aspects of rational number knowledge can be extrapolated from natural numbers. For example, with fractions that have the same denominators, adding together the numerators will give you 


\section{PROFILES OF RATIONAL NUMBER KNOWLEDGE}

the correct numerator for the sum, and numbers with the same number of decimal places can be compared in the same way as natural numbers. Not only can natural number knowledge be a boon for understanding some parts of rational numbers; there are also many types of difficulties students face when reasoning and learning about rational numbers, in contrast to their natural number development. For example, the sheer complexity of fraction notation makes learning fraction procedures more challenging than learning procedures with natural numbers (Behr, Lesh, Post, \& Silver, 1983). However, a large body of research suggests that a substantial part of the difficulties students face when learning about rational numbers stems from a natural number bias (e.g., Ni \& Zhou, 2005).

One of the first instances of this bias, is reasoning about the size of rational numbers (Kainulainen et al., 2017; Stafylidou \& Vosniadou, 2004; Van Hoof, Janssen, et al., 2015). Numerous studies (e.g. Obersteiner, Van Dooren, Van Hoof, \& Verschaffel, 2013; Vamvakoussi et al., 2012; Van Hoof, Lijnen, Verschaffel, \& Van Dooren, 2013) have found that even educated adults show a bias when reasoning about the size of fractions and decimals when their magnitudes are not congruent with their natural number features (e.g., while 6 is smaller than 7, 1/6 is larger than 1/7). Students face a number of particular difficulties with rational number size concepts. First, like educated adults, students often take larger numerators and denominators to represent larger fraction magnitudes (e.g.,Moss, 2005). As well, students often believe that decimal numbers' magnitudes can be determined by the length of the decimal, often equating larger magnitudes with longer decimals (e.g., Durkin \& RittleJohnson, 2015). Research has shown that many students still hold these conceptual misunderstandings even after multiple years of instruction (Van Hoof et al., 2013).

A stronger feature of the natural number bias is the difficulty students have in understanding the dense nature of the set of rational numbers (McMullen, Laakkonen, Hannula-Sormunen, \& Lehtinen, 2015; Vamvakoussi \& Vosniadou, 2004). As with size concepts (e.g. Stafylidou \& Vosniadou, 2004; 


\section{PROFILES OF RATIONAL NUMBER KNOWLEDGE}

Kainulainen et al., 2017), there is evidence that students need to undergo radical conceptual change in order to gain a mathematically correct understanding of the set of rational numbers as being infinitely dense, with an infinite number of numbers - both fractions and decimals - between any two numbers, be them fractions or decimals (Merenluoto \& Lehtinen, 2004; Vamvakoussi et al., 2011; Vamvakoussi \& Vosniadou, 2010). For the most part students show a strong bias in favor of natural number concepts with the set of rational numbers, claiming that there are no, or a limited number of numbers between, for example, $1 / 5$ and 2/5. Once again, educated adults, and even mathematical experts, exhibit signs of the natural number bias when reasoning about the density of rational numbers (Vamvakoussi et al., 2012). The natural number bias with density concepts is also extremely resilient to teaching, though it is rarely explored as an explicit topic in mathematics classrooms in primary or lower secondary schools (McMullen et al., 2015).

Evidence suggests that the natural number bias may be stronger for density than for size concepts (McMullen et al., 2015; Van Hoof, Vandewalle, Verschaffel, \& Van Dooren, 2015), but that these two concepts may also be interconnected (Van Hoof, Janssen, et al., 2015). Students' struggles with understanding both size and density concepts may have the common underlying cause of the natural number bias and thus they can be considered unidimensional (ibid.). There is also evidence to suggest that understanding of size concepts is necessary, but not sufficient for coming to understand density concepts (McMullen et al., 2015). This suggests a common underlying natural number bias that accounts for features of students' difficulties for both size and density concepts. In addition, these previous studies have suggested that the natural number bias is unified across reasoning about both fractions and decimals, even though some students incorrectly consider these two representations of rational numbers to be separate (Vamvakoussi \& Vosniadou, 2010). The present study aims to further 


\section{PROFILES OF RATIONAL NUMBER KNOWLEDGE}

examine the similarities and differences in the patterns of students' fraction and decimal size and density knowledge in both a sample of Finnish and a sample of Flemish students.

\subsection{Conceptual change and latent variable mixture models}

Previously, Vamvakoussi and colleagues (2011) compared Greek and Flemish secondary students' knowledge of density concepts. Results indicated that, in general, patterns of knowledge of density concepts were consistent across the two samples, though Flemish students performed better than the Greek students. These results suggest that the development of density knowledge is somewhat universal since radical conceptual change is needed in both Greek and Flemish students in order to have a mathematically correct concept of the density of the rational number set (see also Vamvakoussi \& Vosniadou, 2010). However, the two-step cluster analysis used by the authors did not allow for a proper comparison of whether the patterns of knowledge between the two samples were similar. The two samples were included in the same cluster analysis and all students were assigned to one of the same five clusters based on this analysis. It is possible, however, that there were structural differences in the patterns of knowledge between the students from Flanders and Greece that were not captured in this analysis. While this analysis provided an overall view of the differences in density knowledge between students, it did not allow for the explicit comparison of the patterns of knowledge between the two sub-samples.

Recently, a number of studies have employed latent variable mixture models in order to examine possible qualitative changes in conceptual knowledge that would be predicted by theories of conceptual change (Edelsbrunner \& Stern, this issue; Flaig et al., this issue; Kainulainen et al., 2017; Schneider \& Hardy, 2013; Straatmeier, van der Maas, \& Jansen, 2008). In particular, the radical restructuring of knowledge predicted by the framework theory of conceptual change (Vosniadou, 2014) has previously 


\section{PROFILES OF RATIONAL NUMBER KNOWLEDGE}

been examined through the use of latent variable mixture models, such as latent class and latent transition analyses (for an overview see Hickendorff et al., this issue). These types of analyses have been employed with concepts of sinking and floating (Edelsbrunner \& Stern, this issue; Schneider \& Hardy, 2013), astronomy (Straatemeier et al., 2008), rational number size (Kainulainen et al., 2017), and human memory processes (Flaig et al., this issue). In these cases, the claim is that there are not only quantitative differences in the amount of correct knowledge students have, but there may be also qualitative differences in the structure and types of knowledge students have, which are not typically captured using continuous or sum scores. The use of these types of latent variable mixture models allows researchers to capture among other things, intermediate states of understanding, which differ both from each other and from scientifically or mathematically correct concepts in qualitative ways.

For example, Schneider and Hardy (2013) found that intermediate levels of understanding of concepts of sinking and floating could be found that did not differ in the number of correct answers the students gave, but had qualitative differences in the patterns of responses that impacted their later development towards a scientifically correct concept. As well, Kainulainen and colleagues (2017) found that students' concepts of rational number size shifted from (1) coherent natural number dependent concepts, through (2) incoherent, intermediate phases, characterized by different synthetic concepts of number, in which natural and rational number concepts are used in inconsistent manners, and into (3) coherent, mathematically-correct concepts of rational number size. This study indicated that latent variable mixture models were useful for identifying the qualitative differences in students' rational number knowledge. These qualitative differences are especially relevant to distinguish between different intermediate phases in which overall performance as measured by a sum score may have indicated similar levels of knowledge, whereas Kainulainen and colleagues' findings suggest differing levels of later success across these intermediate classes. However, this study only examined knowledge 


\section{PROFILES OF RATIONAL NUMBER KNOWLEDGE}

of size concepts in a single educational context, through the use of sum scores, which cannot provide a detailed view on patterns of knowledge. A more complex method using latent variable mixture models allows for the direct comparison of the structure of response patterns across groups (Geiser, Lehmann, \& Eid, 2006). For the first time, the present study will employ such a multigroup latent class analysis (LCA) in order to examine whether students have similar patterns of knowledge with rational number size and density concepts in both the sample of Finnish students and the sample of Flemish students participating in the present study.

\subsection{The present study}

International comparisons can provide specific evidence for the relative level of universality in the development of skills and knowledge (Torbeyns et al., 2015; Vamvakoussi et al., 2011). Importantly, these studies are particularly effective at providing evidence in support of consistency in development, since inconsistencies can be due to multiple factors, such as overall socio-economic factors, teaching traditions, or curricula. So, if similarities can be observed in international comparisons despite those factors, one may argue that there is a relative level of universality in the development of rational number knowledge.

Previously, multiple studies have examined the structure of the natural number bias in students' reasoning about rational number size and density concepts. However, these studies only involve samples from a single educational context (e.g. Kainulainen et al., 2017). The fundamental claim of many researchers is that responses that rely on a natural number bias, while culturally bound, are due to factors that are common to many cultures. In particular, that the disconnects between some features of natural and rational numbers are inherently difficult to manage. As such, recent research found that density conceptual knowledge follows similar patterns in both Greek and Flemish students 


\section{PROFILES OF RATIONAL NUMBER KNOWLEDGE}

(Vamvakoussi et al., 2011). To the best of our knowledge, no previous study has attempted to examine the universality of size concepts or the universality of how size and density rational number knowledge interact during the early learning of rational numbers.

The predominance of natural numbers in both everyday activities and the mathematics classroom in many countries (Andres, Di Luca, \& Pesenti, 2008) leads to the question of whether responses which may be influenced by a natural number bias manifest themselves in Finnish and Flemish students in a similar way. We expect that the overall patterns of students' knowledge of rational number size and density concepts will be fairly similar in both sub-samples, making it possible to identify a single model which encompasses both these Finnish and these Flemish students' patterns of knowledge on items measuring conceptual knowledge of the size and density of rational numbers.

If this is the case, it would indicate that differences between the sub-samples are more due to the level of overall performance than due to differences in the structural make-up of the patterns of knowledge across the different sub-samples (Collins \& Lanza, 2010b; Geiser et al., 2006). While the

present study cannot assure that the structure of rational number knowledge would be universal in all contexts, given the relative similarity of the educational contexts of the two counties (i.e. both high income, high educational attainment, high performance on international comparison tests), it may at least be possible to disprove such universality. In that way, it can be said that if major structural differences exist between two such samples, the universality of rational number size and density knowledge should be called into question. Thus, comparing two relatively similar educational contexts, such as Finland and Flanders, provides an important starting point for moving towards an understanding of the relative universality, or not, of rational number knowledge.

\section{Method}




\section{PROFILES OF RATIONAL NUMBER KNOWLEDGE}

\subsection{Participants}

During one time point in two parallel longitudinal studies examining rational number development (see also, Kainulainen et al., 2017; Van Hoof et al., 2016), 258 Finnish students (136 female) from two different schools and 353 Flemish students (171 female) from five different schools in Grades 4, 5, and 6 completed a test on rational number conceptual knowledge in the Spring term. Table 1 describes the grade level distribution of the Finnish and Flemish students. For the Finnish sub-sample the mean ages were: Grade $4-10$ years, 6 months ( $\mathrm{SD}=4.7$ months); Grade $5-11$ years, 7 months $(\mathrm{SD}=5.2$ months); Grade $6-12$ years 5 months ( $\mathrm{SD}=4.2$ months). For the Flemish sub-sample the mean ages were: Grade $4-10$ years, 5 months ( $\mathrm{SD}=5.9$ months); Grade $5-11$ years, 3 months $(\mathrm{SD}=4.9$ months); Grade $6-12$ years, 5 months ( $\mathrm{SD}=5.4$ months). The Finnish sample was typical of the Finnish urban environment, with students from lower-middle to middle class backgrounds and diverse ethnic heritage (roughly $80 \%$ ethnically Finnish background). The Flemish sample was drawn from schools in predominantly lower-middle to middle class neighborhoods. Data collection was according to the ethical guidelines of the corresponding institutions. 
PROFILES OF RATIONAL NUMBER KNOWLEDGE

Table 1 . Grade by country distribution of participants

\begin{tabular}{llll}
\hline \multicolumn{5}{c}{ Grade } & & \\
& 4 & 5 & 6 \\
\hline Finland & 87 & 78 & 93 \\
& $(34 \%)$ & $(30 \%)$ & $(36 \%)$ \\
Flanders & 148 & 96 & 109 \\
& $(42 \%)$ & $(27 \%)$ & $(31 \%)$ \\
Total & 235 & 174 & 202 \\
& $(38 \%)$ & $(29 \%)$ & $(33 \%)$ \\
\hline
\end{tabular}

\subsection{Fraction and Decimal Instruction in Finland and Flanders}

Fraction and decimal instruction in Finland and Flanders follows a similar pattern in terms of the order of introduction of instruction about the size and density of rational numbers. Students had fraction and decimal instruction covering basic topics such as learning about the representations of rational numbers, including the size of fractions and decimals, basic arithmetic computation with fractions and decimals, and number line estimation. Throughout $3^{\text {rd }}$ and $4^{\text {th }}$ grade, Finnish and Flemish students have been given instruction in understanding the size of both fractions and decimals. Through $6^{\text {th }}$ grade, the Finnish and Flemish students do not have any direct instruction on the density of rational numbers, although they are only indirectly confronted with concepts of density when learning about other concepts with rational numbers, such as the decimal number notation system and finding common denominators.

\subsection{Measure}

Participants completed a measure of their rational number knowledge in a normal classroom environment (Martinie, 2007; Stafylidou \& Vosniadou, 2004; Van Hoof et al., 2015). Items measured conceptual knowledge of the size and density of rational numbers (for a detailed analysis of the reliability and validity of these items see McMullen et al., 2015, and Van Hoof et al., 2016; Cronbach's alphas $=.89$ ). There were altogether fourteen items in which the natural number features of the items 


\section{PROFILES OF RATIONAL NUMBER KNOWLEDGE}

were incongruent with the, mathematically correct, rational number features, so that, for example, the smaller magnitude rational number was made up of larger component numbers (e.g. $5 / 10<4 / 7$, while 5 and 10 are greater than 4 and 7). There were seven size items: three fraction comparison items (e.g., Circle the larger fraction. If the numbers are equal, circle both: $5 / 8 ; 4 / 3)$, one fraction ordering item (Put the numbers in order from largest to smallest: 4/7; 2/6; 5/10), two decimal comparison items (e.g., Circle the larger decimal. If the numbers are equal, circle both: $0.3 ; 0.30)$ and one decimal ordering item (Put the numbers in order from the smallest to the largest: 3.682; 3.2; 3.84). Size items were marked as correct if the participant selected the correct fraction/decimal or put all three fractions/decimals in the correct order. Overall, there were four fraction size items and three decimal size items.

There were seven density items with the same structure ("Are there any fractions/decimals between $\mathrm{X}$ and $\mathrm{Y}$ ? If so, how many). Four items were open-ended and required the participants to fillin an answer (two fraction and two decimal items) while three items were multiple-choice (two fraction and one decimal) in which the students could choose from four possible responses (No numbers/a few [given] numbers/infinite numbers/infinite numbers including fractions and decimals). Open-ended density items were scored as correct if participants acknowledged that there were a substantial number of numbers between the two given, indicating at least a basic understanding that there are an infinite number of numbers between any two fractions or decimals. Multiple-choice density items were scored as correct if the participants selected the last two choices on the multiple choice question ("There are an infinite number of numbers [including fractions or decimals]"). Overall, there were four fraction density items and three decimal density items. 


\section{PROFILES OF RATIONAL NUMBER KNOWLEDGE}

\subsection{Analysis}

In order to examine the nature of the group level differences between the two sub-samples a multigroup LCA was conducted in Mplus Version 7.4 (Muthén \& Muthén, 1998-2016.). The LCAs were conducted based on the participants' responses to the fourteen items of the rational number test. In order to most closely align the models with students' response patterns, the probability of a correct response for each item was used to determine the most likely characteristics of each class. LCA procedure aims to produce classes with as little variation within a class and as much variation between classes as is possible based on the number of classes that are defined. For each LCA models are defined and estimated using by starting with a since class solution and increasing the number of classes in the model by one (Collins \& Lanza, 2010a). The final model for an LCA (i.e., how many classes/cluster are there) is chosen based on a mix of statistical indicators (BIC, likelihood ratio tests) and extant theoretical considerations (Nylund, Asparouhov, \& Muthén, 2007). BIC values indicate goodness of fit, with a lower value indicating a more appropriate fit. Entropy values approaching 1 suggest more certainty in the estimation, with values above .6 considered sufficient. Due to the large sample size, both the Vuong-Lo-Mendell-Rubin Likelihood Ratio Test (VLMR) and the Parametric Bootstrapped Likelihood Ratio Test (BLRT) were used. In these tests, a significant result suggests that the tested model is more appropriate than the model with one fewer class.

In order to determine which is the most appropriate model when there is also a "known class" in the present study the country of the students - multigroup LCA is conducted in three steps (Eid, Langeheine, \& Diener, 2003; Kankaraš, Vermunt, \& Moors, 2010): (1) LCA models are defined for each country separately to determine if there are the same number of classes in each sub-sample, (2) the response patterns are examined to determine if they are the same in each sub-sample (i.e., do the Finnish and Flemish students have the same probability to get the same answers correct?), and (3) the 


\section{PROFILES OF RATIONAL NUMBER KNOWLEDGE}

group membership patterns are compared across sub-samples (i.e., are there the same percentage of Finnish and Flemish students in the identified classes?). The fundamental analytic strategy is to test increasingly unconstrained models in comparison with more constrained models to determine how similar the two sub-samples are, with a deference to more constrained and parsimonious models. Newer, less constrained models are compared pairwise with the preceding model using the SatorraBentler (SB) Chi-square Difference Test (Satorra \& Bentler, 2000).

\section{Results}

\subsection{Descriptives and overall country level differences}

Univariate ANOVAs (see Table 2) revealed that the Flemish students performed better than the Finnish students on items measuring decimal size knowledge, $F(1,572)=54.85, p<.001$ and decimal density knowledge, $F(1,572)=9.54, p=.002$, but there were no differences in fraction size or density knowledge (see Table 2). These results suggest that there are at least some differences in the level of the Finnish and Flemish students' performance on these items.

Table 2

Overall Means (and Standard Deviations) for the proportion of correct responses for the four components of rational number knowledge for Finnish $(n=258)$ and Flemish $(n=353)$ students.

\begin{tabular}{lllll}
\hline & Fraction Size & Decimal Size & $\begin{array}{l}\text { Fraction } \\
\text { Density }\end{array}$ & Decimal Density \\
\hline Finnish & .53 & .54 & .27 & .28 \\
& $(.38)$ & $(.43)$ & $(.23)$ & $(.24)$ \\
Flemish & .58 & .79 & .26 & .35 \\
& $(.34)$ & $(.33)$ & $(.29)$ & $(.29)$ \\
Overall & .56 & .69 & .26 & .32 \\
& $(.36)$ & $(.40)$ & $(.27)$ & $(.27)$ \\
\hline
\end{tabular}




\section{PROFILES OF RATIONAL NUMBER KNOWLEDGE}

\subsection{Multigroup LCA}

First, LCA models were defined for Finnish and Flemish students separately in order to determine if there were the same number of classes in each sub-sample. Statistical tests of model fit can be found in Table 3. Based on the low BIC values and statistical significance of the VLRM test, these results suggest that in the Finnish sub-sample a four- or five-class model would be most appropriate and in the Flemish sub-sample a four-class model would be most appropriate.

-Insert Table 3 about here-

As the next step, a multigroup LCA was estimated with both Finnish and Flemish students included in the same model. In the prior step it was revealed that either the four- or five-class model appeared to be the most appropriate in both the sub-samples. However, when comparing these models across the sub-samples it is apparent that there are fundamental differences in the overall success rates, which already have been noted based on the analyses from section 3.1. These differences suggest that the additional class of the five class model may be necessary in order to appropriately capture the different response patterns across groups that may appear (Collins \& Lanza, 2010b). In particular, a class of students who were successful on most items of both size and density was seen in the Flemish sub-sample, whereas the three other classes aligned with similar classes in the Finnish sub-sample. Thus, while in the separate sub-samples four classes may have been sufficient to capture the breadth of variation, when looking at the two sub-samples together there appeared to be the need for a fifth class 


\section{PROFILES OF RATIONAL NUMBER KNOWLEDGE}

to cover the wider range of variation. For this reason, the multigroup LCA was run as a five-class model. $^{1}$

Since the overall performance levels varied between the Finnish and Flemish students, there was no need to run a model with total invariance for both response patterns and class membership. Thus, the multigroup LCA was run first with total invariance for students' response patterns across the subsamples. Such a model assumes that the pattern of students' knowledge of rational numbers is the same between the two sub-samples in that it holds constant item response probabilities for each class across the two sub-samples, but allows for a differing proportion of students to be included in each class. Next a model with partial invariance was estimated, in which those items with the largest differences in patterns of success in the different grades between the sub-samples were not constrained to be equal (i.e. the four open-ended fraction and decimal density items). This allows for the class-level item response probabilities to vary between the two sub-samples on the items that were most likely to vary across sub-samples in some classes, while holding item response probabilities constant within corresponding classes on all other items. Again, class membership assignment was allowed to vary across the two sub-samples, reflecting the variation in overall performance between the two subsamples. Finally, we estimated a model with no measurement invariance, in which no items were constrained to be equal (e.g., response patterns could differ freely across sub-sample classes). Results reveal that the partial invariance model was more appropriate than the total invariance model, SB Chisquare Difference Test $\Delta \chi^{2}(20)=53.97, p<.001$, and the non-invariance model was more appropriate than the partial invariance model, $\Delta \chi^{2}(50)=131.35, p<.001$. Thus the non-invariance model was chosen as the most appropriate model.

\footnotetext{
${ }^{1}$ Additionally, statistical fit indices revealed that the five-class models of the differing invariance levels were always more appropriate than the corresponding four-class models
} 


\section{PROFILES OF RATIONAL NUMBER KNOWLEDGE}

\subsection{A model of non-invariance}

Figures 1a-1e detail the probability of a correct response for each item by LCA class for both of the Finnish and Flemish sub-samples in the non-invariance model (See Appendix for Mplus input syntax). The resulting classes showed a large amount of overlap in the structure of response probabilities, to the extent that the profiles could be described similarly across both the Finnish and the Flemish sub-samples, suggesting that differences between the two sub-samples may be more due to overall levels of success rather than structural differences.

The first class of students (Low: Finn: 40\%; Flem: 16\%) could be described as having a low level of success on all items, with the exception of the second fraction comparison item (i.e., 2/7 vs 5/6). The next class (Partial Size: Finn: 12\%; Flem: $n=16 \%$ ) had success on the decimal size items, but was mostly unsuccessful with all other items. The next class, which was the first class to show consistent levels of one type of knowledge (i.e., size of rational numbers), was also the largest class overall (Size: Finn: 33\%; Flem: 23\%) and had relatively high probability of success on almost all of the size items for both fractions and decimals. Further along in their rational number knowledge was a class which had consistent success with size items, but mixed success with density items, particularly doing well on decimal density items, but not fraction density items (Partial Density: Finn: 10\%; Flem: 25\%). Moderate differences in the probability of getting the open-ended and multiple-choice decimal density items were apparent in the partial density classes. Finally, the class that displayed the highest level of knowledge of rational numbers, was likely to be successful on almost every item (Density: Finn: 4\%;

Flem: 20\%). In this class, there were minor differences in the probability of success on the open-ended fraction density items.

-Insert Figures 1a-1e about here- 


\section{PROFILES OF RATIONAL NUMBER KNOWLEDGE}

The Finnish students did better than their Flemish counterparts on the fraction ordering item. In particular, even those students in the Flemish sub-sample who were successful on all other items, had about an equal chance of getting the fraction ordering item right or wrong. As well, in comparing the Density classes, the Finnish students, while much smaller in number, had a high chance of success on all items, while the Flemish students only had mixed success on the open-ended versions of the fraction density items (Correct response probabilities: Finnish: 92\% vs Flemish: 54\% and Finnish: 100\% vs Flemish: 45\%); the Flemish students in this class do however slightly outperform the Finnish students on the multiple-choice density items. A curiosity of the Partial Density classes is that students in the Finnish sub-sample were much more likely to get the correct answer on the decimal density multiple choice items than their Flemish counterparts (Finnish: 93\%, Flemish: 34\%), while these Finnish students had a lower chance of getting the correct answer on the open-ended decimal density items (Finnish: 61\%, Flemish: 98\%).

\section{Discussion}

Previously, it has been revealed that students' difficulties in understanding the dense nature of the rational number set were fairly similar between Greek and Flemish students, even though Flemish students in general performed better than the Greek students (Vamvakoussi et al., 2011). The present study expands on this work by proposing a structural similarity between Flemish and Finnish students' knowledge of rational number size and density concepts. These results reveal that besides relatively minor differences in the patterns of knowledge the students display, Finnish and Flemish participants' conceptual knowledge followed fairly similar structures. While it was not possible to define a single model which encompassed both the Finnish and Flemish students' patterns of rational number size and density knowledge, the resulting model did show a large overlap in terms of these patterns across the two sub-samples. 


\section{PROFILES OF RATIONAL NUMBER KNOWLEDGE}

Results of the multigroup LCA indicate that the most appropriate method when modelling the Finnish and Flemish participants' rational number knowledge was using no measurement invariance (Eid et al., 2003). However, results reveal that there were only limited differences in the structure of students' rational number knowledge in the Finnish and Flemish sub-samples. For the most part the resultant classes were fairly well matched across the two sub-samples. These findings indicate that it is at least possible that there is some universality in how students progress in their understanding of rational number concepts. That similar classes of students with particular response patterns appear in both a sample of Finnish and a sample of Flemish students suggests that patterns of rational number knowledge may not be entirely dependent on educational contexts.

One advantage in using a multigroup LCA is to explicitly test whether there are differences in the levels and/or structures of knowledge between the sub-samples included in the analysis (Geiser et al., 2006). In contrast to previous studies which modelled students' patterns of knowledge in aggregate (Vamvakoussi et al., 2011), the present study is the first to employ such an explicit test of the structural similarities and differences in rational number knowledge between students from different countries. The results of the present study suggest that the differences in rational number knowledge between these Finnish and Flemish students are more due to overall levels of knowledge rather than structural differences. While the Flemish students were more likely to be in more successful classes, particularly with regard to the Partial Density and Density classes and the Finnish students were more likely to be in the Low class, results did not suggest major structural differences between the two countries.

The present study also provides evidence of what the structure of students' rational number size and density knowledge may look like during the first phase of learning about fractions and decimals. In particular, the results of the present study show that for the most part knowledge of fraction and decimal size and density follows two distinct patterns. 


\section{PROFILES OF RATIONAL NUMBER KNOWLEDGE}

The first relevant pattern is that students' success on most items seems to fall into the extremes, where they are either very likely to get an item correct (or even a sub-set of items, e.g., fraction comparison, decimal density open-ended) or are very unlikely to get that item correct. This suggests that the model is fairly able to create distinct classes of students with similar response patterns (Collins \& Lanza, 2010a; Geiser et al., 2006). A mid-range (e.g., 50\%) chance on items suggests that some students in that class are able to get the item correct, while others are not. Consistent response probabilities around chance for many classes could be an indication that the item is not a strong marker of the underlying knowledge being tested. In the case of the present study, the fraction ordering item both shows inconsistent patterns between the two sub-samples and has fairly poor discriminating power for many of the classes. For example, as a whole the Finnish Size class only had around a 50\% chance of getting the item correct. The procedural difficulty of, and the greater number of potential strategies that could be used on, this item may have caused more measurement error than other items, for example, students listing the answers correctly, but in a reverse order. Future studies should take this potential issue into account.

These extremes also provide further evidence of a strong natural number bias in students' reasoning about rational numbers size and density concepts at this age (Kainulainen et al., 2017; Vamvakoussi \& Vosniadou, 2010). As students show strong consistency in their answers on the subgroup of items, when they get an answer wrong they are also consistent with this. This consistency in wrong responses suggests a deliberate process, most likely in this case using natural number concepts to solve these problems. Since the items were designed specifically to be incongruent with natural and rational number features, these patterns of responses suggests a bias towards natural number features when reasoning about rational numbers for those who are unsuccessful on the tasks. 


\section{PROFILES OF RATIONAL NUMBER KNOWLEDGE}

The second relevant pattern found in the structure of these students' rational number knowledge that is revealing in its consistency across the two sub-samples is the relative success on the decimal items in those students with a partial understanding of the size and density of rational numbers. In both sub-samples, the Partial Size and Partial Density classes were successful on the decimal items for the corresponding concept, but unsuccessful on the fraction items. Corresponding classes with fraction knowledge being high while decimal knowledge is low do not appear in these sub-samples. While it is worth noting that the number of Finnish students in these classes was relatively low, the most appropriate model did still suggest that these classes are relevant for this sample of Finnish students. While previous research has found inter-relations between fraction and decimal knowledge among this age-group (McMullen et al., 2015; Van Hoof, Janssen, et al., 2015), the intermediate levels of "decimal-but-not-fraction" knowledge that appear in these sub-samples suggests that it may be easier to overcome the natural number bias with decimals.

The present study provides the first evidence of the commonality in multiple aspects of rational number knowledge between two distinct groups of students from different countries. While the results suggest future research could pursue this examination further in samples with less commonalities, there are a number of limitations to the present study that should be taken into account in these future studies. First and foremost, it is important to examine such international differences with sub-samples that are explicitly drawn to be nationally representative in order to confirm that there are not substantial sampling effects that influence the results of the present study, including the unbalanced grade distribution between the sub-samples. Next, more detailed information about the actual instruction given to these students regarding these topics is necessary to understand the differences that do appear in the present and any future studies. The relatively similar make-up of these sub-samples' knowledge does not necessitate too detailed an analysis of the curricula that was covered in the students' 


\section{PROFILES OF RATIONAL NUMBER KNOWLEDGE}

classrooms in the current study, but any analysis which wants to explain uncovered differences must take this into account. As well, countries with larger differences between their instruction practices, educational attainment, and cultural context would be interesting to compare (e.g. Torbeyns et al., 2015), considering the relatively similar levels of achievement and culture between the two northern European counties compared in the present study (OECD, 2014).

Previously, Van Hoof and colleagues (2015) have more effectively shown the influence of the natural number bias on students' performance by comparing performance on both congruent (i.e., natural and rational number features are in line with each other, e.g., 1/3 vs 2/3) and incongruent (i.e., natural and rational number features are in conflict with each other, e.g., 1/2 vs 1/3) items. In the present study, only incongruent items were used and, thus, no specific estimation of the strength of the natural number bias in these sub-samples can be made. This opens the possibility that the differences in the levels of knowledge found here between the two sub-samples may be more reflective of a general deficit in rational number understanding or a reflection of more successful overcoming of the natural number bias (e.g. Alibali \& Sidney, 2015). This distinction is subtle, but it must be taken into account if any future studies would like to examine the role of instruction on the natural number bias.

Ultimately, the present study highlights the relative similarity in the structure of these Finnish and Flemish students' patterns of rational number knowledge. The results indicate only relatively minor differences in the overall structure of these knowledge patterns, suggesting that in these samples rational number knowledge expresses in the students in similar ways. In this way, it appears that there are mainly differences in the levels of knowledge of rational numbers these students have. However, the differences that appear in the level of success with rational number concepts between the two countries' samples, particularly with regard to decimal and density knowledge, suggest that more research into the role of instruction in the development of rational number concepts would be 


\section{PROFILES OF RATIONAL NUMBER KNOWLEDGE}

beneficial. Examinations of individual differences in learners' development across time through longitudinal investigations of rational number knowledge may be particularly fruitful.

\section{References}

Alibali, M. W., \& Sidney, P. G. (2015). Variability in the natural number bias: Who, when, how, and why. Learning and Instruction 37, 56-61. doi:10.1016/j.learninstruc.2015.01.003

Andres, M., Di Luca, S., \& Pesenti, M. (2008). Finger counting: The missing tool? Behavioral and Brain Sciences, 31, 642-643. doi:10.1017/S0140525X08005578

Behr, M., Lesh, R., Post, T. R., \& Silver, E. A. (1983). Rational-number concepts. In Lesh, R. (ed.) Acquisition of mathematics concepts and processes (pp. 91-126). Academic Press: New York.

Collins, L. M., \& Lanza, S. T. (2010a). Latent Class and Latent Transition Analysis: With applications in the social, behavioral, and health sciences. Hoboken, NJ: Wiley.

Collins, L. M., \& Lanza, S. T. (2010b). Multiple-Group Latent Transition Analysis and Latent Transition Analysis with Covariates. Latent Class and Latent Transition Analysis, 41, 225-265. doi:10.1002/9780470567333.ch8

DeWolf, M., Bassok, M., \& Holyoak, K. J. (2015). From rational numbers to algebra: Separable contributions of decimal magnitude and relational understanding of fractions. Journal of Experimental Child Psychology, 133, 1-13. doi:10.1016/j.jecp.2015.01.013

Durkin, K., \& Rittle-Johnson, B. (2015). Measuring misconceptions: Revealing changing decimal fraction knowledge. Learning and Instruction, 37, 21-29. doi:10.1016/j.learninstruc.2014.08.003

Eid, M., Langeheine, R., \& Diener, E. (2003). Comparing typological structures across cultures by Multigroup Latent Class Analysis: A primer. Journal of Cross-Cultural Psychology, 34, 195-210. doi:10.1177/0022022102250427

Geiser, C., Lehmann, W., \& Eid, M. (2006). Separating "rotators" from "nonrotators" in the Mental Rotations Test: A Multigroup Latent Class Analysis. Multivariate Behavioral Research, 41, 261293. doi:10.1207/s15327906mbr4103_2

Handel, M. J. (2016). What do people do at work? Journal for Labour Market Research. doi:10.1007/s12651-016-0213-1

Kainulainen, M., McMullen, J., \& Lehtinen, E. (2017). Early developmental trajectories towards the concept of rational numbers. Cognition and Instruction, 35. doi:10.1080/07370008.2016.1251287

Kankaraš, M., Vermunt, J. K., \& Moors, G. (2010). Testing for Measurement Invariance with Latent Class Analysis. In Davidov, E., Schmidt, P., Billiet, J. (eds.) Crosscultural analysis methods and applications (pp. 359-384). Psychology Press:New York. 


\section{PROFILES OF RATIONAL NUMBER KNOWLEDGE}

Martinie, S. L. (2007). Middle school rational number knowledge. Kansas State University Press.

McMullen, J., Laakkonen, E., Hannula-Sormunen, M., \& Lehtinen, E. (2015). Modeling the developmental trajectories of rational number concept(s). Learning and Instruction, 37, 14-20. doi:10.1016/j.learninstruc.2013.12.004

Merenluoto, K., \& Lehtinen, E. (2004). Number concept and conceptual change: towards a systemic model of the processes of change. Learning and Instruction, 14, 519-534. doi:10.1016/j.learninstruc.2004.06.016

Moss, J. (2005). Pipes, tubes, and beakers: new approaches to teaching the rational-number system. In M. S. Dovovan \& J. D. Bransford (Eds.) How students learn: Mathematics in the classroom (pp. 309-349). The National Academies Press, Washington D.C.

Muthén, L. K., \& Muthén, B. O. (1998-2016). Mplus user's guide. Seventh Edition. Los Angeles, CA: Muthén and Muthén.

Ni, Y., \& Zhou, Y.-D. Y. (2005). Teaching and learning fraction and rational numbers: The origins and implications of whole number bias. Educational Psychologist, 40, 27-52. doi:10.1207/s15326985ep4001_3

Nunes, T., \& Bryant, P. (2008). Rational numbers and intensive quantities: Challenges and insights to pupils' implicit knowledge. Anales De Psicologia, 24, 262-270.

Nylund, K. L., Asparouhov, T., \& Muthén, B. O. (2007). Deciding on the number of classes in Latent Class Analysis and Growth Mixture Modeling: A Monte Carlo simulation study. Structural Equation Modeling: A Multidisciplinary Journal, 14, 535-569. doi:10.1080/10705510701575396

Obersteiner, A., \& Tumpek, C. (2016). Measuring fraction comparison strategies with eye-tracking. ZDM - Mathematics Education, 48, 255-266. Doi:10.1007/s11858-015-0742-Z

Obersteiner, A., Van Dooren, W., Van Hoof, J., \& Verschaffel, L. (2013). The natural number bias and magnitude representation in fraction comparison by expert mathematicians. Learning and Instruction, 28, 64-72. doi:10.1016/j.learninstruc.2013.05.003

OECD (2014). PISA 2012 results in focus. Programme for International Student Assessment, 1-44. doi:10.1787/9789264208070-en

Satorra, A., \& Bentler, P. (2000). A scaled difference chi-square test statistic for moment structure analysis. Psychometrika, 66, 507-514. doi:10.1007/bf02296192

Schneider, M., \& Hardy, I. (2013). Profiles of inconsistent knowledge in children's pathways of conceptual change. Developmental Psychology, 49, 1639-49. doi:10.1037/a0030976

Siegler, R. S., Duncan, G. J., Davis-Kean, P. E., Duckworth, K., Claessens, A., Engel, M., ... Chen, M. (2012). Early predictors of high school mathematics achievement. Psychological Science, 23, 691-697. doi:10.1177/0956797612440101 
Stafylidou, S., \& Vosniadou, S. (2004). The development of students' understanding of the numerical value of fractions. Learning and Instruction, 14, 503-518. doi:10.1016/j.learninstruc.2004.06.015

Straatemeier, M., van der Maas, H. L. J., \& Jansen, B. R. J. (2008). Children's knowledge of the earth: a new methodological and statistical approach. Journal of Experimental Child Psychology, 100, 276-96. doi:10.1016/j.jecp.2008.03.004

Torbeyns, J., Schneider, M., Xin, Z., \& Siegler, R. S. (2015). Bridging the gap: Fraction understanding is central to mathematics achievement in students from three different continents. Learning and Instruction, 37, 5-13. doi:10.1016/j.learninstruc.2014.03.002

Vamvakoussi, X., Christou, K. P., Mertens, L., \& Van Dooren, W. (2011). What fills the gap between discrete and dense? Greek and Flemish students' understanding of density. Learning and Instruction, 21, 676-685. doi:10.1016/j.learninstruc.2011.03.005

Vamvakoussi, X., Van Dooren, W., \& Verschaffel, L. (2012). Naturally biased? In search for reaction time evidence for a natural number bias in adults. The Journal of Mathematical Behavior, 31, 344-355. doi:10.1016/j.jmathb.2012.02.001

Vamvakoussi, X., \& Vosniadou, S. (2004). Understanding the structure of the set of rational numbers: a conceptual change approach. Learning and Instruction, 14(5), 453-467. doi:10.1016/j.learninstruc.2004.06.013

Vamvakoussi, X., \& Vosniadou, S. (2010). How many decimals are there between two fractions? Aspects of secondary school students , understanding of rational numbers and their notation. Cognition and Instruction, 28, 181-209. doi:10.1080/07370001003676603

Van Hoof, J., Degrande, T., Verschaffel, L., \& Van Dooren, W. (2016). The relation between learners' spontaneous focusing on quantitative relations and their rational number knowledge. Studia Psychologica, 58, 156-170.

Van Hoof, J., Janssen, R., Verschaffel, L., \& Van Dooren, W. (2015). Inhibiting natural number knowledge in fourth graders: towards a comprehensive test instrument. ZDM - Mathematics Education, 47. doi:10.1007/s11858-014-0650-7

Van Hoof, J., Lijnen, T., Verschaffel, L., \& Van Dooren, W. (2013). Are secondary school students still hampered by the natural number bias? A reaction time study on fraction comparison tasks. Research In Mathematics Education, 15, 154-164. doi:10.1080/14794802.2013.797747

Van Hoof, J., Vandewalle, J., Verschaffel, L., \& Van Dooren, W. (2015). In search for the natural number bias in secondary school students' interpretation of the effect of arithmetical operations. Learning and Instruction, 37, 30-38. doi:10.1016/j.learninstruc.2014.03.004

Vosniadou, S. (2014). Examining cognitive development from a conceptual change point of view: The framework theory approach. European Journal of Developmental Psychology, 11. doi:10.1080/17405629.2014.921153 
PROFILES OF RATIONAL NUMBER KNOWLEDGE

\section{Appendix}

Mplus input syntax for final model:

TITLE: $\quad$ MultiGroup LCA, non-invariance, 5 classes

DATA: $\quad$ FILE IS data.dat;

VARIABLE: NAMES $=$ ID Country

Size1-7 Dens1-7F;

USEVARIABLES ARE

Size1-7 Dens1-7F;

auxiliary is id;

CATEGORICAL ARE

Size1-7 Dens1-7F;

MISSING ARE ALL(-999);

KNOWNCLASS $=\operatorname{cc}($ Country $=1$ Country $=2)$;

CLASSES $=\mathrm{c}(5) \operatorname{cc}(2)$;

ANALYSIS: $\quad$ TYPE $=$ MIXTURE;

STARTS $=1000,100$

MODEL: $\quad \%$ OVERALL\%

cc\#1 ON C\#1 C\#2 C\#3 C\#4;

OUTPUT: $\quad$ SAMPSTAT TECH1 TECH8 TECH10; 